\title{
Two-Twistor Space, Commuting Composite Minkowski Coordinates and Particle Dynamics
}

\author{
A. Bette ${ }^{1)}$, J. Lukierski ${ }^{2)}$ and C. Miquel-Espanya ${ }^{3)}$ \\ 1) Royal Institute of Technology \\ S-151 81 Södertälje, Sweden \\ 2) Institute for Theoretical Physics, \\ University of Wrocław, \\ pl. M. Borna 9, 50-204 Wrocław, Poland \\ 3) Departament de Física Teòrica i IFIC, \\ Universitat de València, \\ Dr. Moliner 50, 46100 Burjassot (València), Spain
}

\begin{abstract}
We employ the modification of the basic Penrose formula in twistor theory, which allows to introduce commuting composite space-time coordinates. It appears that in the course of such modification the internal symmetry $S U(2)$ of two-twistor system is broken to $U(1)$. We consider the symplectic form on two-twistor space, permitting to interpret its 16 real components as a phase-space. After a suitable change of variables such a two-twistor phase space is split into three mutually commuting parts, describing respectively the standard relativistic phase space ( 8 degrees of freedom), the spin sector (6 degrees of freedom) and the canonical pair angle-charge describing the electric charge sector (2 degrees of freedom). We obtain a geometric framework providing a twistor-inspired 18-dimensional extended relativistic phase space $\mathcal{M}^{18}$. In such a space we propose the action only with first class constraints, describing the relativistic particle characterized by mass, spin and electric charge.
\end{abstract}

\section{Introduction}

The choice of basic geometric variables that describe the dynamics at the most elementary level is an important issue extensively discussed in mathematical physics as well as in fundamental interactions theory. In standard relativistic $D=4$ theory we assume that the basic geometry is described by the Minkowski space-time coordinates $x_{\mu}=\left(\vec{x}, x_{0}=c t\right)$. 
There are two ways of extending the notion of classical Minkowski space-time:

i) One adds additional geometric degrees of freedom, e.g. the anticommuting Grassman variables in supersymmetric theory or additional commuting continuous or discrete coordinates. In principle the replacement of elementary point particles by strings can be also described as the extension of Minkowski space by infinite set of auxiliary coordinates describing Fourier modes of an extended object. In all these approaches the space-time coordinates remain elementary.

ii) One can consider the space-time geometry as a derived notion, with composite space-time coordinates. Because the most elementary representation of the Lorentz algebra is spinorial ${ }^{1}$, natural candidates for new elementary coordinates are Lorentz spinors. Taking into consideration that the mass can be considered as a dynamical effect, these elementary spinorial coordinates describing primary kinematics should describe the geometry of massless world with basic conformal invariance. In such a way we arrive at the notion of twistors (see e.g. [1-5]) - fundamental representations of the conformal algebra - as describing the coordinates of primary geometry.

In four dimensions $(D=4)$ the conformal algebra is $S U(2,2)=\overline{S O(4,2)}$, and its fundamental twistor representation $T^{4}=\left(Z_{1}, Z_{2}, Z_{3}, Z_{4}\right)$ is complex. We define the twistor space as a fourdimensional complex metric space $T^{4}=\left(c^{4}, h\right)$, where the Hermitean metric $h$ has the signature $(+,+,-,-)$. Choosing

$$
h=\left(\begin{array}{cc}
0 & I_{2} \\
I_{2} & 0
\end{array}\right)
$$

one can represent a twistor by a pair of $D=4$ Weyl $\operatorname{spinors}^{2}(\alpha, \beta=1,2 ; A=1,2,3,4)$

$$
Z_{A}=\left(\omega^{\alpha}, \bar{\pi}_{\dot{\beta}}\right), \quad \bar{Z}_{A}=\left(Z_{A}\right)^{*}=\left(\bar{\omega}^{\dot{\alpha}}, \pi_{\beta}\right) .
$$

More explicitly, the $S U(2,2)$ norm can be written as follows

$$
\langle T, T\rangle=Z_{A} h^{A B} \bar{Z}_{B}=Z_{A} \bar{Z}^{A}=\omega^{\alpha} \pi_{\alpha}+\bar{\omega}^{\dot{\alpha}} \bar{\pi}_{\dot{\alpha}} .
$$

The link with space-time coordinates is obtained by imposing the Penrose incidence relations

$$
\omega^{\alpha}=i z^{\alpha \dot{\beta}} \bar{\pi}_{\dot{\beta}}, \quad \bar{\omega}^{\dot{\alpha}}=-i \bar{z}^{\beta \dot{\alpha}} \pi_{\beta},
$$

where

$$
z^{\alpha \dot{\beta}}=\frac{1}{2}\left(\sigma_{\mu}\right)^{\alpha \dot{\beta}} z^{\mu}, \quad \bar{z}^{\beta \dot{\alpha}}=\frac{1}{2}\left(\bar{\sigma}_{\mu}\right)^{\beta \dot{\alpha}} \bar{z}^{\mu},
$$

describe complex Minkowski coordinates. From (4) follows that the complex Minkowski coordinates parametrize two-planes in $T^{4}$, i.e.

$$
z_{\mu} \in G_{4 ; 2}(c)=\frac{S U(2,2)}{S(U(2) \times U(2))} .
$$

\footnotetext{
${ }^{1}$ By most elementary representation we mean that all other irreducible representation can be obtained by tensoring procedure.

${ }^{2}$ We define $\omega^{\alpha}=\epsilon^{\alpha \beta} \omega_{\beta}, \bar{\omega}^{\dot{\alpha}}=\epsilon^{\dot{\alpha} \dot{\beta}} \bar{\omega}_{\dot{\beta}}, \omega_{\alpha}=\omega^{\beta} \epsilon_{\beta \alpha}, \bar{\omega}_{\dot{\alpha}}=\bar{\omega}^{\dot{\beta}} \epsilon_{\dot{\beta} \dot{\alpha}}$ and $\epsilon^{\alpha \beta} \epsilon_{\beta \gamma}=-\delta_{\gamma}^{\alpha}, \epsilon^{\dot{\alpha} \dot{\beta}} \epsilon_{\dot{\beta} \dot{\gamma}}=-\delta_{\dot{\gamma}}^{\dot{\alpha}}$, where $\epsilon^{\alpha \beta}=\epsilon_{\alpha \beta}=\left(\begin{array}{cc}0 & -1 \\ 1 & 0\end{array}\right)=-\epsilon^{\dot{\alpha} \dot{\beta}}=-\epsilon_{\dot{\alpha} \dot{\beta}}$.
} 
Any such plane is parametrized by a pair of nonparallel twistors

$$
Z_{A ; i}=\left(\omega_{; i}^{\alpha}, \bar{\pi}_{\dot{\beta} ; i}\right) \quad \bar{Z}_{A}^{; i}=\left(Z_{A ; i}\right)^{*}=\left(\bar{\omega}^{\dot{\alpha} ; i}, \pi_{\beta}^{; i}\right) \quad i=1,2
$$

where we observe that the complex-conjugated spinors are contravariant in the internal $U(2)$ index space, i.e. $\left(\pi_{\alpha ; i}\right)^{*}=\bar{\pi}_{\dot{\alpha}}{ }^{; i}$ etc. In such a way the $U(2)$-invariant norm we denote $A_{; i}\left(A_{; i}\right)^{*}=A_{; i} \bar{A}^{; i}{ }^{3}$ Writing down the relation (41) for two twistors

$$
\omega_{; i}^{\alpha}=i z^{\alpha \dot{\beta}} \bar{\pi}_{\dot{\beta} ; i}, \quad \bar{\omega}^{\dot{\alpha} ; i}=-i \bar{z}^{\beta \dot{\alpha}} \pi_{\beta}^{; i},
$$

one gets as the solution of (8) the complex composite Minkowski space-time coordinates

$$
z^{\alpha \dot{\beta}}=x^{\alpha \dot{\beta}}+i y^{\alpha \dot{\beta}}=\frac{i}{f} \omega^{\alpha ; i} \bar{\pi}_{; i}^{\dot{\beta}},
$$

where $A^{; i}=\epsilon^{i j} A_{; j}$, the fourvectors $x_{\mu}, y_{\mu}$ are real, and

$$
f=\bar{\pi}_{; 1}^{\dot{\alpha}} \bar{\pi}_{\dot{\alpha} ; 2}=\frac{1}{2} \bar{\pi}_{\dot{\alpha} ; i} \bar{\pi}_{; j}^{\dot{\alpha}} \epsilon^{i j}=\frac{1}{2} \bar{\pi}_{\dot{\alpha} ; i} \bar{\pi}^{\dot{\alpha} ; i}, \quad \bar{f}=\frac{1}{2} \pi_{\alpha ; i} \pi^{\alpha ; i},
$$

consistently with the numerical equality $\epsilon_{i j}=\epsilon^{i j}$. From the relation (9) one can show that in order to embed the real Minkowski coordinates in twistor spaces one should consider pairs of twistors $T_{1}, T_{2}$ which span a null 2-plane, i.e.

$$
t_{i}^{j}=\left\langle T_{i}, T_{j}\right\rangle=Z_{A ; i} h^{A B} \bar{Z}_{B ;}^{j}=0 .
$$

In such a case one gets from (8) that $y_{\mu}=0$, i.e. the formula (9) describes real Minkowski coordinates.

The Hermitean metric $h$ generates the $S U(2,2)$-invariant symplectic two-form $\Omega$. Using (11) and (17) one can write

$$
\begin{aligned}
\Omega & =i d Z_{A ; i} \wedge d \bar{Z}_{B}^{; i} h^{A B} \\
& =i\left(d \omega^{\alpha}{ }_{; i} \wedge d \pi_{\alpha}^{; i}+d \bar{\pi}_{\dot{\alpha} ; i} \wedge d \bar{\omega}^{\dot{\alpha} ; i}\right)
\end{aligned}
$$

and the Liouville one form $\Theta$, satisfying the relation $\Omega=d \Theta$, looks as follows

$$
\Theta=\frac{i}{2}\left(\omega_{; i}^{\alpha} d \pi_{\alpha}^{; i}+\bar{\pi}_{\dot{\alpha} ; i} d \bar{\omega}^{\dot{\alpha} ; i}-H . C\right)
$$

It appears that the two-form (12) defines fundamental twistorial Poisson brackets (TPB) and the Liouville one-form (13) should be important for the construction of dynamical Lagrangean and Hamiltonian models in twistor space.

The problem which we firstly consider in Sect. 2 is the proper notion of composite space-time in twistor space. If we use the formula (9) it was shown [6] that the real composite Minkowski space coordinates $x_{\mu}=R e z_{\mu}$ are noncommuting. Following [7. 8. 9] we introduce the modification of the standard Penrose formula (9) which leads to

\footnotetext{
${ }^{3}$ We define by analogy with the footnote 2 that $A^{; i}=\epsilon^{i j} A_{; j}, A_{; i}=A^{; j} \epsilon_{j i}$ where $\epsilon^{i j}=\left(\begin{array}{cc}0 & -1 \\ 1 & 0\end{array}\right)=\epsilon_{i j}$.
} 
commuting composite space-time coordinates $X_{\mu}$. Further in Sect. 3 by considering the nonlinear transformations of sixteen real coordinates in two-twistor space we introduce an enlarged 18-dimensional relativistic phase space $\mathcal{M}^{18}$ with three mutually commuting sectors

i) Relativistic phase-space $\left(X_{\mu}, P_{\mu}\right)$

ii) Spinorial complex phase space $\left(\eta_{\alpha}, \sigma_{\alpha}, \bar{\eta}_{\dot{\alpha}}, \bar{\sigma}_{\dot{\alpha}}\right)$

iii) Electric charge phase space $(e, \phi)$

One can prove that the equivalence with the two-twistor space (17) implies the imposition of two constraints in the spinorial complex phase space [10.

In Sect. 4 we consider in the extended phase space $\mathcal{M}^{18}=\left(X_{\mu}, P_{\mu}, \eta_{\alpha}, \bar{\eta}_{\dot{\alpha}}, \sigma^{\alpha}\right.$, $\left.\bar{\sigma}_{\dot{\alpha}}, e, \phi\right)$ a free particle model. In [10] we considered such a model with two geometric second class constraints, and three physical first class constraints defining physical quantities: mass, spin and charge. It appears however that the classical Dirac brackets, obtained in the process of elimination of the second class constraints, are very difficult to quantize in a consistent way (Jacobi identities!). In this paper we propose an alternative model in $\mathcal{M}^{18}$, with the two second class constraints proposed in 10 replaced by one geometric first class constraints. In such a model with four first class constraints (one geometric, three physical) the quantization is straightforward.

In the last Section we shall present the concluding remarks.

\section{Standard relativistic phase space from two-twistor geometry}

The two-twistor symplectic form (12) implies the following nonzero fundamental Poisson brackets

$$
\begin{aligned}
& \left\{\pi_{\alpha}^{; i}, \omega_{; j}^{\beta}\right\}=i \delta_{\alpha}^{\beta} \delta_{j}^{i}, \\
& \left\{\bar{\pi}_{\dot{\alpha} ; i}, \bar{\omega}^{\dot{\beta} ; j}\right\}=-i \delta_{\dot{\alpha}}^{\dot{\beta}} \delta_{i}^{j} .
\end{aligned}
$$

After quantization one obtains from (14) the basic twistorial canonical commutation relations (TCCR)

$$
\begin{aligned}
& {\left[\widehat{\omega}_{; j}^{\beta}, \widehat{\pi}_{\alpha}^{; i}\right]=\hbar \delta_{\alpha}^{\beta} \delta_{j}^{i},} \\
& {\left[\widehat{\bar{\omega}}^{\dot{\beta} ; j}, \widehat{\bar{\pi}}_{\dot{\alpha} ; i}\right]=-\hbar \delta_{\dot{\alpha}}^{\dot{\beta}} \delta_{i}^{j} .}
\end{aligned}
$$

Using the relation (9) one can calculate the TPB of the real composite Minkowski coordinates $x_{\mu}$. One gets [6]

$$
\left\{x_{\mu}, x_{\nu}\right\}=-\frac{1}{m^{4}} \epsilon_{\mu \nu \rho \tau} W^{\rho} P^{\tau}
$$


where

$$
m^{2}=2|f|^{2}=2\left|\frac{1}{2} \bar{\pi}_{\dot{\alpha} ; i} \bar{\pi}^{\dot{\alpha} ; i}\right|^{2}=\frac{1}{2}\left|\bar{\pi}_{\dot{\alpha} ; i} \bar{\pi}^{\dot{\alpha} ; i}\right|^{2}=P_{\mu} P^{\mu}
$$

and $(r=1,2,3)$

$$
\begin{aligned}
P^{\alpha \dot{\beta}} & =\pi^{\alpha ; i} \bar{\pi}_{; i}^{\dot{\beta}}, & P^{\mu}=\sigma^{\mu}{ }_{\alpha \dot{\beta}} P^{\alpha \dot{\beta}}, \\
W^{\alpha \dot{\beta}} & =\pi^{\alpha ; i}\left(\tau^{r}\right)_{i}^{j} \bar{\pi}_{; j}^{\dot{\beta}} t_{r}, & W^{\mu}=\sigma_{\alpha \dot{\beta}}^{\mu} W^{\alpha \dot{\beta}},
\end{aligned}
$$

where $(a=0,1,2,3)$

$$
t_{i}^{j}=\left(\tau^{a}\right)_{i}^{j} t_{a}=\left\langle T_{i}, T_{j}\right\rangle=Z_{A ; i} \bar{Z}^{A ; j}
$$

and $\left(\tau^{0}\right)_{i}^{j}=\left(\begin{array}{ll}1 & 0 \\ 0 & 1\end{array}\right)$ and $\left(\tau^{r}\right)_{i}^{j}$ describe three Pauli isospin matrices. The internal isospin symmetry is represented by the following $s u(2) \otimes u(1)$ Poisson algebra brackets $(r, s, u=$ $1,2,3)$

$$
\left\{t_{r}, t_{s}\right\}=\epsilon_{r s u} t_{u}, \quad\left\{t_{0}, t_{r}\right\}=0,
$$

as it follows from (14) and (20).

Using the relation (18) one can extended the TPB (16) by the following two relations:

$$
\begin{gathered}
\left\{P_{\mu}, x_{\nu}\right\}=\eta_{\mu \nu}, \\
\left\{P_{\mu}, P_{\nu}\right\}=0 .
\end{gathered}
$$

Replacing the TPB (14) and (22, 23) by TCCR (15) one gets the quantized relativistic phase space $\left(\widehat{x}_{\mu}, \widehat{P}_{\mu}\right)$ with noncommuting composite Minkowski coordinates $\widehat{x}_{\mu}$. Such noncommutativity in the presence of nonvanishing spin $\left(W_{\mu} \neq 0\right)$ can be traced back to earlier considerations by Souriau [1] and Casalbuoni [12. Indeed, the composite fourvector (19) can be identified with the Pauli-Lubanski vector in arbitrary relativistic frame. It is orthogonal, as it should be, to the composite fourmomentum (18)

$$
P_{\mu} W^{\mu}=0,
$$

and in the rest system ${ }^{4} P_{\mu}=(m, 0,0,0)$ one can write the noncommutativity relations of quantized composite Minkowski coordinates as folows: $\left(\widehat{x}_{\mu}=\left(\widehat{x}_{k}, \widehat{x}_{0}=c \widehat{t}\right)\right.$

$$
\left[\widehat{x}_{k}, \widehat{x}_{l}\right]=-i \frac{\hbar}{m^{2}} \epsilon_{k l m} \widehat{S}_{m}
$$

where $\widehat{W}_{k}=m \widehat{S}_{k}$ and as follows from (19)

$$
\left[\widehat{S}_{k}, \widehat{S}_{l}\right]=i \hbar \epsilon_{k l m} \widehat{S}_{m}
$$

\footnotetext{
${ }^{4}$ In quantized systems such description implies the consideration of eigenstates of the four-momentum operator $\widehat{P}_{\mu}$.
} 
In this lecture we would like to consider the composite relativistic phase space $\left(X_{\mu}, P_{\mu}\right)$, satisfying the standard TPB

$$
\begin{aligned}
\left\{X_{\mu}, X_{\nu}\right\} & =\left\{P_{\mu}, P_{\nu}\right\}=0 \\
\left\{X_{\mu}, P_{\nu}\right\} & =\eta_{\mu \nu}
\end{aligned}
$$

In such a case one has to change the definition of composite Minkowski coordinates by the modification of the standard definition (9), which we redefine as follows [7, 8, 9, 10]

$$
\begin{aligned}
z^{\alpha \dot{\beta}} \longrightarrow Z^{\alpha \dot{\beta}} & =z^{\alpha \dot{\beta}}+\Delta z^{\alpha \dot{\beta}}=X^{\alpha \dot{\beta}}+i Y^{\alpha \dot{\beta}} \\
& =z^{\alpha \dot{\beta}}+i\left(t_{1}-i t_{2}\right) \frac{\pi^{\alpha ; 1} \bar{\pi}_{; 2}^{\dot{\beta}}}{|f|^{2}}
\end{aligned}
$$

or

$$
\begin{aligned}
X^{\alpha \dot{\beta}} & =x^{\alpha \dot{\beta}}+\Delta x^{\alpha \dot{\beta}}=x^{\alpha \dot{\beta}}-\frac{1}{2|f|^{2}}\left[t_{1} \pi^{\alpha ; i}\left(\tau_{2}\right)_{i}{ }^{j} \bar{\pi}_{; j}^{\dot{\beta}}-t_{2} \pi^{\alpha ; i}\left(\tau_{1}\right)_{i}{ }^{j} \bar{\pi}_{; j}^{\dot{\beta}}\right] \\
& =x^{\alpha \dot{\beta}}-\frac{1}{2|f|^{2}} \epsilon_{3 r s} t_{r} \pi^{\alpha ; i}\left(\tau_{s}\right)_{i}{ }^{j} \bar{\pi}_{; j}^{\dot{\beta}} .
\end{aligned}
$$

One can show that the TPB of the composite coordinates (29) are given by the relation (27) i.e. after quantization we obtain commuting composite Minkowski coordinate. We see from the relations (29) that the commutative coordinates $X^{\mu}=\left(\sigma^{\mu}\right)_{\alpha \dot{\beta}} X^{\alpha \dot{\beta}}$ distinguish a third direction in the isospace $O(3) \simeq S U(2)$ i.e. break the isospin symmetry from $O(3)$ to $O(2)$.

\section{$3 \quad$ Extended relativistic phase space from two-twistor space.}

Let us consider the symplectic 2-form (13) and insert the formula (91) for the composite complex Minkowski coordinates. Using the $S U(2)$-covariant notation ${ }^{5}$ one gets

$$
\Theta=\pi_{\alpha}^{; i} \bar{\pi}_{\dot{\beta} ; i} d x^{\alpha \dot{\beta}}+i y^{\alpha \dot{\beta}}\left(\pi_{\alpha}^{; i} d \bar{\pi}_{\dot{\beta} ; i}-\bar{\pi}_{\dot{\beta} ; i} d \pi_{\alpha}^{; i}\right)
$$

Using the formula for the imaginary part of the complex four-vector $z_{\mu}$ one gets

$$
t^{i}{ }_{j}=-2 y^{\alpha \dot{\beta}} \pi_{\alpha}^{; i} \bar{\pi}_{\dot{\beta} ; j},
$$

\footnotetext{
${ }^{5}$ The advantage of such a notation has been pointed out to us by S. Fedoruk. In such a framework the "half-twistors" $\pi_{\alpha ; i}, \bar{\pi}_{\dot{\alpha} ; i}$ can be treated as a pair of $S l(2 ; C)$ harmonic spinors (see e.g. [13, 14] 15]) with particular normalization.
} 


$$
y^{\alpha \dot{\beta}}=-\frac{1}{2|f|^{2}} t_{i}^{j} \pi_{; j}^{\alpha ; i} \bar{\pi}^{\dot{\beta}}
$$

One gets using

$$
\begin{array}{r}
\bar{\pi}_{; i}^{\dot{\alpha}} \bar{\pi}_{\dot{\alpha} ; j}=-\epsilon_{i j} f, \quad \pi^{\alpha ; i} \pi_{\alpha}^{; j}=-\epsilon^{i j} \bar{f}, \\
\bar{\pi}_{\dot{\alpha} ; i} \bar{\pi}_{\dot{\beta}}^{; i}=\epsilon_{\dot{\alpha} \dot{\beta}} f, \quad \pi_{\alpha ; i} \pi_{\beta}^{; i}=\epsilon_{\alpha \beta} \bar{f},
\end{array}
$$

that

$$
\begin{aligned}
y^{\alpha \dot{\beta}} \pi_{\alpha}{ }^{; k} & =\frac{1}{2 f} t_{i}^{j} \bar{\pi}_{; j}^{\dot{\beta}} \epsilon^{i k}, \\
y^{\alpha \dot{\beta}} \bar{\pi}_{\dot{\beta} ; k} & =\frac{1}{2 \bar{f}} t_{i}^{j} \pi^{\alpha ; i} \epsilon_{j k} .
\end{aligned}
$$

Substituting in (30) the formula (18) and using (33) one gets $\left(\left(t^{i}{ }_{j}\right)^{*}=t_{i}{ }^{j}\right)$

$$
\begin{aligned}
\Theta & =P_{\mu} d x^{\mu}+i\left[-\frac{1}{2 \bar{f}} t_{i}^{j} \pi^{\alpha ; i} \epsilon_{j k} d \pi_{\alpha}^{; k}+\frac{1}{2 f} t_{i}^{j} \bar{\pi}_{; j}^{\dot{\beta}} \epsilon^{i k} d \bar{\pi}_{\dot{\beta} ; k}\right] \\
& =P_{\mu} d x^{\mu}+i\left[\frac{1}{2 \bar{f}} t_{i}^{j} \pi^{\alpha ; i} d \pi_{\alpha ; j}+\frac{1}{2 f} t_{i}^{j} \bar{\pi}_{; j}^{\dot{\beta}} d \bar{\pi}_{\dot{\beta}}^{; i}\right] .
\end{aligned}
$$

Let us observe that ${ }^{6}$

$$
\begin{aligned}
& \bar{\pi}_{; i}^{\dot{\beta}} d \bar{\pi}_{\dot{\beta} ; j}=\bar{\pi}_{; i i}^{\dot{\beta}} d \bar{\pi}_{\dot{\beta} ; j]}+\bar{\pi}_{; i}^{\dot{\beta}} d \bar{\pi}_{\dot{\beta} ; j)}, \\
& \pi^{\beta ; i} d \pi_{\beta}^{; j}=\pi^{\beta ;[i} d \pi_{\beta}^{; j]}+\pi^{\beta ;(i} d \pi_{\beta}^{; j)},
\end{aligned}
$$

and observe that

$$
\begin{aligned}
\bar{\pi}_{;[i}^{\dot{\beta}} d \bar{\pi}_{\dot{\beta} ; j]} & =-\frac{1}{2} \epsilon_{i j} d f \\
\pi^{\beta ;[i} d \pi_{\beta}^{; j]} & =-\frac{1}{2} \epsilon^{i j} d \bar{f} .
\end{aligned}
$$

We obtain

$$
\Theta=P_{\mu} d x^{\mu}+\frac{i}{2} t_{i}^{j}\left(\frac{1}{\bar{f}} \pi^{\alpha ;(i} d \pi_{\dot{\alpha}}{ }^{; k)} \epsilon_{k j}+\frac{1}{f} \bar{\pi}_{;(j}^{\dot{\beta}} d \bar{\pi}_{\dot{\beta} ; k)} \epsilon^{i k}\right)+\frac{i}{4} t_{k}^{k} d \ln \left(\frac{\bar{f}}{f}\right) .
$$

\footnotetext{
${ }^{6}$ We define $A_{(i} B_{j)}=\frac{1}{2}\left(A_{i} B_{j}+A_{j} B_{i}\right)$ and $A_{[i} B_{j]}=\frac{1}{2}\left(A_{i} B_{j}-A_{j} B_{i}\right)$.
} 
In the symplectic prepotential (34) the space-time coordinates after quantization are noncommutating. In order to introduce symplectic form with commuting space-time coordinates $X_{\mu}$ one should use the formulae (28)

$$
\begin{aligned}
P_{\mu} d x^{\mu} & =P_{\mu} d X^{\mu}-P_{\alpha \dot{\beta}} d\left(\Delta x^{\alpha \dot{\beta}}\right) \\
& =P_{\mu} d X^{\mu}-\epsilon_{3 r s} P_{\alpha \dot{\beta}} d\left(\frac{-1}{2|f|^{2}} t_{r} \pi^{\alpha ; i}\left(\tau_{s}\right)_{i}{ }^{j} \bar{\pi}_{; j}^{\dot{\beta}}\right) .
\end{aligned}
$$

Using the formula (18) one obtains

$$
\begin{aligned}
P_{\mu} d x^{\mu} & =P_{\mu} d X^{\mu}+P_{\alpha \dot{\beta}} \frac{1}{2|f|^{2}} \epsilon_{3 r s} t_{r}\left(\tau_{s}\right)_{i}{ }^{j} d\left(\pi^{\alpha ; i} \bar{\pi}_{; j}^{\dot{\beta}}\right) \\
& =P_{\mu} d X^{\mu}+\epsilon_{3 r s} t_{r}\left(\tau_{s}\right)_{i}{ }^{j}\left[\frac{1}{2 \bar{f}} \pi_{\alpha ; j} d \pi^{\alpha ; i}-\frac{1}{2 f} \bar{\pi}_{\dot{\beta}}^{; i} d \bar{\pi}_{; j}^{\dot{\beta}}\right]
\end{aligned}
$$

Substituting (39) in (37) one gets $(r=1,2,3)$

$$
\Theta=P_{\mu} d X^{\mu}+\frac{i}{f} t_{r}\left(\tau_{r}\right)_{1}^{j} \bar{\pi}_{; j}^{\dot{\alpha}} d \bar{\pi}_{\dot{\alpha} ; 2}-\frac{i}{\bar{f}} t_{r}\left(\tau_{r}\right)_{j}{ }^{1} \pi^{\alpha ; j} d \pi_{\dot{\alpha}}{ }^{2}+\frac{i}{2}\left(t_{0}+t_{3}\right)\left(\frac{d \bar{f}}{\bar{f}}-\frac{d f}{f}\right)
$$

or

$$
\Theta=P_{\mu} d X^{\mu}-i\left(\bar{\sigma}_{; 1}^{\dot{\alpha}} d \bar{\pi}_{\dot{\alpha} ; 2}-\sigma^{\alpha ; 1} d \pi_{\alpha}^{; 2}\right)+e d \phi,
$$

where $(r=1,2,3)$

$$
\begin{aligned}
\sigma^{\alpha ; i} & =-\frac{1}{\bar{f}} t_{r}\left(\tau_{r}\right)_{j}{ }^{i} \pi^{\alpha ; j}, \\
\bar{\sigma}_{; i}^{\dot{\alpha}} & =-\frac{1}{f} t_{r}\left(\tau_{r}\right)_{i}{ }^{j} \bar{\pi}_{; j}^{\dot{\alpha}}, \\
e & =t_{0}+t_{3}, \\
\phi & =\frac{i}{2} \ln \frac{\bar{f}}{f},
\end{aligned}
$$

implying

$$
d \phi=\frac{i}{2}\left(\frac{d \bar{f}}{\bar{f}}-\frac{d f}{f}\right) .
$$

We see therefore that the second part of the symplectic form (41) is the second rank fixed spinor in the internal space, and represents in the internal three-space $(S U(2) \simeq$ $O(3))$ the direction $a_{1}+i a_{2}$. The third part, describing the electric charge sector, indicates 
the direction $a_{0}+a_{3}$ ( $a_{0}$ is a scalar), i.e. the $S U(2)$ symmetry is also broken. It can be mentioned that the formula (42) for the electric charge recalls the Gell-Mann-Nishijima formula $Q=I_{3}+\frac{Y}{2}$, where in $Y$ is a scalar from the point of view of the internal $S U(2)$ isospin symmetry.

Let us point out that the 18 variables $\left(X_{\mu}, P_{\mu}, \pi^{\alpha ; 2}, \bar{\pi}_{\dot{\alpha} ; 2}, \sigma^{\alpha ; 1}, \bar{\sigma}_{\dot{\alpha} ; 1}, e, \phi\right)$ as composites of two-twistor space coordinates are not independent. On can show from (18) and (42) (see also [10] ${ }^{7}$ ) that they satisfy the following three kinematical constraints:

$$
\begin{aligned}
& R_{1}=\sigma_{\alpha}{ }^{; 1} P^{\alpha \dot{\beta}} \bar{\sigma}_{\dot{\alpha} ; 1}-\mathbf{t}^{2}=0, \quad \mathbf{t}^{2}=\left(t_{1}\right)^{2}+\left(t_{2}\right)^{2}+\left(t_{3}\right)^{2} \\
& R_{2}=\pi_{\alpha}{ }^{; 2} P^{\alpha \dot{\beta}} \bar{\pi}_{\dot{\beta} ; 2}-\frac{1}{2} P^{2}=0, \\
& R_{3}=\pi_{\alpha}{ }^{; 2} \sigma^{\alpha ; 1}-\bar{\pi}_{\dot{\alpha} ; 2} \bar{\sigma}_{; 1}^{\dot{\alpha}}=0,
\end{aligned}
$$

where (we recall that $f=\sqrt{\frac{P^{2}}{2}} e^{i \phi}$ )

$$
\begin{aligned}
t_{1}{ }^{2}=t_{1}-i t_{2} & =-\frac{1}{\bar{f}} \pi_{\alpha}{ }^{; 2} P^{\alpha \dot{\beta}} \bar{\sigma}_{\dot{\beta} ; 1}, \\
t_{2}{ }^{1}=t_{1}+i t_{2} & =-\frac{1}{f} \sigma_{\alpha}{ }^{; 1} P^{\alpha \dot{\beta}} \bar{\pi}_{\dot{\beta} ; 2}, \\
t_{1}{ }^{1}-t_{2}{ }^{2}=2 t_{3} & =\pi^{\alpha ; 2} \sigma_{\alpha}{ }^{; 1}-\bar{\pi}_{\dot{\alpha} ; 2} \bar{\sigma}_{; 1}^{\dot{\alpha}} .
\end{aligned}
$$

The constraints (45)-(47) reduce the 18 independent variables in (41) to 16 degrees of freedom in two-twistor space because only two of them are independent.

The symplectic form (41) implies the following canonical Poisson brackets (CPB):

$$
\begin{gathered}
\left\{X_{\mu}, X_{\nu}\right\}=0, \quad\left\{P_{\mu}, P_{\nu}\right\}=0, \quad\left\{P_{\mu}, X_{\nu}\right\}=\eta_{\mu \nu} \\
\left\{\pi_{\alpha}^{; 2}, \sigma^{\beta ; 1}\right\}=i \delta_{\alpha}^{\beta}, \quad\left\{\bar{\pi}_{\dot{\alpha} ; 2}, \bar{\sigma}^{\dot{\beta}}{ }^{\dot{\beta}}\right\}=-i \delta_{\dot{\alpha}}^{\dot{\beta}} \\
\{e, \phi\}=1 .
\end{gathered}
$$

Let us observe that the three constraints $\left(R_{1}, R_{2}, R_{3}\right)$ have the following $\mathrm{CPB}$ :

$$
\begin{aligned}
& \left\{R_{1}, R_{2}\right\}=-2 i \sigma_{\alpha}{ }^{; 1} P^{\alpha \dot{\beta}} \bar{\sigma}_{\dot{\beta} ; 1}, \\
& \left\{R_{2}, R_{3}\right\}=2 i \pi_{\alpha}{ }^{2} P^{\alpha \dot{\beta}} \bar{\pi}_{\dot{\beta} ; 2} \\
& \left\{R_{1}, R_{3}\right\}=-i R_{2} \cdot R_{3} .
\end{aligned}
$$

\footnotetext{
${ }^{7}$ We should mention that the constraints $R_{1} \cdots R_{6}$ are linear combinations of the constraints given in [10
} 
We suplement them with the following additional three physical constraints:

$$
\begin{aligned}
& R_{4}=\mathbf{t}^{2}-s(s+1)=0, \\
& R_{5}=P^{2}-m^{2}=0, \\
& R_{6}=e-e_{0}=0 .
\end{aligned}
$$

Using the canonical PB (see (49a-c)) one gets the following relations: $(A, B=3,4,5,6)$

$$
\left\{R_{A}, R_{B}\right\}=0
$$

We see therefore that one can consider the set $\left(R_{3}, R_{4}, R_{5}, R_{6}\right)$ as four first class constraints.

The constraints $R_{4}=R_{5}=0$ can be interpreted as determining the numerical value of the mass operator $P^{2}$ and the isospin square $\mathbf{t}^{2}$. If we observe further that [6, 10,

$$
\mathbf{t}^{2}=-\frac{1}{2|f|^{2}} W_{\alpha \dot{\beta}} W^{\alpha \dot{\beta}}=-\frac{1}{P^{2}} W^{2}
$$

where $W^{2} \equiv W_{\alpha \dot{\beta}} W^{\alpha \dot{\beta}}$ describes the square of the composite Pauli-Lubański fourvector, we see that one can identify $\mathbf{t}^{2}$ with the relativistic spin square Casimir of the Poincare algebra.

It should be added that the constraints (51a-c) can be supplemented with another relation determining the projection of isospin on the third axis.

\section{Relativistic particles with mass, spin and electric charges in extended space-time.}

Let us consider the 18 coordinates $\left(X_{\mu}, P_{\mu}, \eta^{\alpha} \equiv \pi^{\alpha ; 2}, \bar{\eta}_{\dot{\alpha}} \equiv \bar{\pi}_{\dot{\alpha} ; 2}, \sigma^{\alpha} \equiv \sigma^{\alpha ; 1}, \bar{\sigma}_{\dot{\alpha}} \equiv\right.$ $\left.\bar{\sigma}_{\dot{\alpha} ; 1}, e, \phi\right)$ occuring in the symplectic one-form (41) as primary ones. They shall be restricted by four constraints $R_{3}=\cdots=R_{6}=0$. We propose the following action with four constraints introduced through Lagrangian multipliers:

$$
S=\int d \tau \mathcal{L}=\int d \tau\left[P_{\mu} \dot{X}^{\mu}+i\left(\sigma^{\alpha} \dot{\eta}_{\alpha}-\bar{\sigma}^{\dot{\alpha}} \dot{\bar{\eta}}_{\dot{\alpha}}\right)+e \dot{\phi}+\sum_{i=2}^{i=6} \lambda_{i} R_{i}\right],
$$

where $R_{i}(i=3, \ldots, 6)$ are given by the formulae (47), (51a-c). Using (49a-c) one can show that all four constraints are first class. One can quantize the model by canonical quantization of the PB (49a-c). Using the standard quantization rule $(i \hbar\{a, b\} \leftrightarrow[\hat{a}, \hat{b}])$ one gets the following canonical commutators: 


$$
\begin{array}{cc}
{\left[\hat{X}_{\mu}, \hat{X}_{\nu}\right]=\left[\hat{P}_{\mu}, \hat{P}_{\nu}\right]=0,} & {\left[\hat{P}_{\mu}, \hat{X}_{\nu}\right]=i \hbar \eta_{\mu \nu},} \\
{\left[\hat{\sigma}^{\alpha}, \hat{\eta}_{\beta}\right]=\hbar \delta_{\beta}^{\alpha},} & {\left[\hat{\bar{\sigma}}^{\dot{\alpha}}, \hat{\bar{\eta}}_{\dot{\beta}}\right]=-\hbar \delta_{\dot{\beta}}^{\dot{\alpha}},} \\
{[\hat{e}, \hat{\phi}]=i \hbar .}
\end{array}
$$

We introduce the Schrödinger representation in the extended momentum space $\mathcal{P}_{k}=$ $\left(P_{\mu}, \eta_{\alpha}, \bar{\eta}_{\dot{\alpha}}, \phi\right)(\mathrm{k}=1, \ldots, 9)$ as follows

$$
\begin{aligned}
\hat{X}_{\mu}=-i \hbar \frac{\partial}{\partial P^{\mu}}, \quad \hat{\sigma}_{\alpha} & =\hbar \frac{\partial}{\partial \eta^{\alpha}}, \quad \hat{\bar{\sigma}}_{\dot{\alpha}}=-\hbar \frac{\partial}{\partial \bar{\eta}^{\dot{\alpha}}}, \\
\hat{e} & =i \hbar \frac{\partial}{\partial \phi} .
\end{aligned}
$$

In our quantized model the dynamics is characterized by the following four wave equations describing the wave function $\Psi\left(\mathcal{P}_{k}\right) \equiv \Psi\left(P_{\mu}, \eta_{\alpha}, \bar{\eta}_{\dot{\alpha}}, \phi\right)$

$$
\begin{aligned}
R_{3}=0: \quad & \left(\eta_{\alpha} \frac{\partial}{\partial \eta^{\alpha}}+\bar{\eta}_{\dot{\alpha}} \frac{\partial}{\partial \bar{\eta}^{\dot{\alpha}}}\right) \Psi\left(\mathcal{P}_{k}\right)=0, \\
R_{4}=0: \quad & {\left[\eta_{\alpha} \bar{\eta}_{\dot{\alpha}} \frac{\partial}{\partial \eta_{\alpha}} \frac{\partial}{\partial \bar{\eta}_{\dot{\alpha}}}\right.} \\
& +\frac{1}{P^{2}} P_{\alpha \dot{\alpha}} P_{\beta \dot{\beta}}\left(\eta^{\alpha} \epsilon^{\dot{\beta} \dot{\alpha}} \frac{\partial}{\partial \eta_{\beta}}+\bar{\eta}^{\dot{\beta}} \epsilon^{\alpha \beta} \frac{\partial}{\partial \bar{\eta}_{\dot{\alpha}}}+2 \eta^{\alpha} \bar{\eta}^{\dot{\beta}} \frac{\partial}{\partial \eta_{\beta}} \frac{\partial}{\partial \bar{\eta}_{\dot{\alpha}}}\right) \\
& \left.+\frac{s(s+1)}{\hbar^{2}}\right] \Psi\left(\mathcal{P}_{k}\right)=0, \\
R_{5}=0: \quad & \left(P^{2}-m^{2}\right) \Psi\left(\mathcal{P}_{k}\right)=0, \\
R_{6}=0: \quad & \left(\frac{\partial}{\partial \phi}+\frac{i}{\hbar} e_{0}\right) \Psi\left(\mathcal{P}_{k}\right)=0 .
\end{aligned}
$$

The set (57atd) describes the quantized first class constraints. If we add fifth constraint $R_{4}^{\prime}=t_{3}-m_{3}=0$, where $-s \leq m_{3} \leq s$, we obtain the description of a massive relativistic particle with spin $s$, isospin projection $m_{3}$ on the third axis and the electric charge $e_{0}$. One can observe that the differential form of the Lorentz-invariant third component of isospin $t_{3}$

$$
t_{3}=\frac{1}{2}\left(\eta_{\alpha} \frac{\partial}{\partial \eta_{\alpha}}-\bar{\eta}_{\dot{\alpha}} \frac{\partial}{\partial \bar{\eta}_{\dot{\alpha}}}\right)
$$

corresponds to the twistorial helicity formula for massless particles [1]-4] employed recently in Witten's tensorial string theory [16

It should be stressed that in distinction with [10] in our model we do not have the constraints $R_{1}=R_{2}=0$, i.e. our phase space can not be identified with the two-twistorial 
phase space. Let us observe, however, that the conditions $R_{1}=0$ or $R_{2}=0$ can be obtained as gauge-fixing conditions for the gauge freedom generated by the constraint $R_{3}$, and viceversa, the transition from second to first class constraints is obtained by so-called gauge unfixing procedure (see e.g. [17, 18]).

More detailed discussion of the quantization of the model (54) will be given in a subsequent publication [19.

\section{$5 \quad$ Final Remarks}

In the present lecture it is described the "physical basis" for two-twistor phase space and considered the particle models based on the symplectic form (41).

We stress that for the description of massive relativistic particles with spin we consider both relativistic phase space coordinates $\left(X_{\mu}, P_{\mu}\right)$ as composite (compare e.g. with [20] where the coordinates $X_{\mu}$ are elementary) as well as we do not introduce any additional degrees of freedom besides two-twistor space (compare with [21, 22, where additional so-called index spinor was introduced).

In comparison with the results given in [10] we presented here the following two new aspects:

i) In the process of introduction of a "physical basis" in two-twistor space, defining the enlarged coomposite relativistic phase space $\mathcal{M}^{18}$, we exhibited explicitely the covariance and the breaking of the internal symmetry $S U(2)$.

ii) We introduced the particle model only inspired by the two-twistor space geometry with entirely first class constraints. If we wish to link the phase space of our model with two-twistor manifold and composite twistor formulae for $P_{\mu}, X_{\mu}, \eta_{\alpha}, \bar{\eta}_{\dot{\alpha}}, \sigma_{\alpha}, \bar{\sigma}_{\dot{\alpha}}, e$ and $\phi$ one should consider the gauge-fixed version of the model. In such a way will appear the second class constraints, considered in [10].

In this lecture there is presented only the model in four dimensions $(D=4)$. It appears that one can extend our considerations to the pair of super-twistors (see e.g. [23. 24] ) and consider the corresponding superparticle models with mass and superspin. Other generalization consists in the extension of our discussion to other dimensions $D$ (e.g. $D=10$ or $D=11$; see e.g. [24]-[26]).

\section{Acknowledgments}

We would like to thank J.A. de Azcárraga, S. Fedoruk and A. Frydryszak for valuable remarks. One of the authors (J.L.) would like to acknowledge the support of the KBN grant 1 P03B 01828 and one of us (C.M-E.) wishes to thank the Spanish M.E.C. for his research grant. 


\section{References}

[1] R. Penrose, Int. Journ. Theor. Phys. 1, 61 (1968); Twistor theory, its aims and achievements, in Quantum Gravity, C.J. Isham et al. (eds.), Clarendon, Oxford 1975, p. 268-407.

[2] R. Penrose and M.A. MacCallum, Phys. Rep. C6, 241 (1972).

[3] Z. Perjés, Rep. Math. Phys. 12, 193 (1977).

[4] L.P. Hughston, Twistors and Particles, Lecture Notes in Physics 97, Springer Verlag, Berlin (1979).

[5] P.A. Tod, Rep. Math. Phys. 11, 339 (1977).

[6] A. Bette, J. Math. Phys. 25, 2456 (1984).

[7] A. Bette and S. Zakrzewski, J. Phys. A30, 195 (1997).

[8] A. Bette and S. Zakrzewski, Massive relativistic particles with spin and the two twistor phase space, in Proc. XIIth Workshop on Soft Physics-Hadrons 96, Crimea. Pub. Nat. Acad. of Sci. of Ukraine, Kiev 1996, p. 336, hep-th/0404024.

[9] A. Bette, Twistors, special relativity, conformal symmetry and minimal coupling, hep-th/0402150.

[10] A. Bette, J. de Azcárraga, J. Lukierski and C. Miquel-Espanya, Phys. Lett. B595, 491 (2004).

[11] J.M. Souriau, Structure des systèmes dynamiques, Dunod, Paris (1970).

[12] R. Casalbuoni, Nuovo Cim. 33A, 389 (1976).

[13] I. Bandos, Sov. J. Nucl. Phys. 51, 906 (1990).

[14] I. Bandos and A. Zheltukhin, Class. Quant. Grav. 12, 609 (1995).

[15] V. Zima and S. Fedoruk, Theor. Math. Phys. 102, 305 (1995).

[16] E. Witten, Comm. Math. Phys. 252, 189 (2004).

[17] L. Faddeev, S. Shatashvili, Phys. Lett. B167, 225 (1986).

[18] A.S. Vytheeswaran, Ann. Phys. (NY) 236, 297 (1994).

[19] A. Bette, J. de Azcárraga, J. Lukierski and C. Miquel-Espanya, in preparation.

[20] S.L. Lyakhovich, A.A. Sharapov and K.M. Shekter, Nucl. Phys. b537, 640 (1999); Int. J. Mod. Phys. A15, 4287 (2000). 
[21] S. Fedoruk, V.G. Zima, Mod. Phys. Lett. A15, 2281 (2000).

[22] S. Fedoruk, V.G. Zima, hep-th/0308154 hep-th/0401064.

[23] A. Ferber, Nucl. Phys. B132, 55 (1978).

[24] I. Bandos, J. Lukierski and D. Sorokin, Phys. Rev. D61, 045002 (2000).

[25] I. Bars, Phys. Lett. B483, 248 (2000).

[26] I. Bandos, J. A. de Azcárraga, J. M. Izquierdo and J. Lukierski, Phys. Lett. 86, 4451 (2001). 